\title{
IMPACTO DA PROCURA DE SENSACÕES SEXUAIS NOS COMPORTAMENTOS DE VIOLÊNCIA NO NAMORO
}

\author{
Paula Nelas \\ Escola Superior de Saúde, Instituto Politécnico de Viseu, UICISA: E - IPV/ESSV \\ pnelas@gmail.com \\ Cláudia Chaves \\ Escola Superior de Saúde, Instituto Politécnico de Viseu, CI\&DEI \\ claudiachaves21@gmail.com \\ Emília Coutinho \\ Escola Superior de Saúde, Instituto Politécnico de Viseu, UICISA: E - IPV/ESSV \\ ecoutinhoessv@gmail.com
}

Recepción Artículo: 27 agosto 2021

Admisión Evaluación: 07 septiembre 2021

Informe Evaluador 1: 12 septiembre 2021

Informe Evaluador 2: 13 septiembre 2021

Aprobación Publicación: 15 septiembre 2021

\section{RESUMO}

Enquadramento: A violência no namoro tem revelado uma elevada preocupação e reconhecimento, tanto a nível cientííico, como social, pelo impacto que tem na saúde física e mental. Objetivos: Determinar se as sensações sexuais influenciam as práticas e comportamentos de violência no namoro. Participantes: A amostra é constituída por 820 estudantes do ensino superior (média de idade de 20.48 anos ( $\mathrm{Dp}=2.51$ ). Método: É um estudo quantitativo, descrito-correlacional e transversal. 0 protocolo de investigação foi um questionário que permitiu caracterizar a amostra a nível sociodemográfico, académico, afetivo e sexual. Incluímos a Escala de Práticas e Comportamentos de Violência no Namoro (Dixe, Rodrigues, Freire, Rodrigues, Fernandes \& Dias (2010) e a Escala de Procura de Sensações Sexuais (validada para a população portuguesa por Santos, Ferreira, Carvalho \& Ferreira, 2017). Resultados: Trata-se de uma amostra maioritariamente feminina (69.4\%). Relativamente às práticas e comportamentos de violência no namoro verificamos, nas dimensões em análise, valores máximos de 80,70, para a violência psicológica, 91,67 para a violência stalking e violência sexual e 90.48 para a violência física. Os resultados da árvore de decisão revelam que a procura de sensações sexuais, é a variável que melhor prediz o grau de violência do namoro. Conclusões: Uma vez que a violência nas relações de intimidade causa danos no desenvolvimento físico, psicológico, sexual, reprodutivo, social, académico e profissional, com efeitos na saúde e no bem-estar das pessoas, quer a curto, quer a médio e a longo prazo, propomos ações de sensibilização em contexto escolar promovidas por peritos e debatidas entre pares.

Palavras-chave: violência; namoro; estudantes; ensino superior; sensações sexuais 


\section{IMPACTO DA PROCURA DE SENSAÇÕES SEXUAIS NOS COMPORTAMENTOS DE VIOLÊNCIA NO NAMORO}

\section{ABSTRACT}

Impact of sexual sensation seeking on dating violence behaviors. Background: Dating violence has shown a high concern and recognition, both scientifically and socially, for the impact it has on physical and mental health. Objectives: Determine whether sexual feelings influence dating violence practices and behaviors. Participants: The sample consisted of 820 university students (mean age 20,48 years, $\mathrm{Sd}=2.51$ ). Method: It is a quantitative, descriptive-correlational and cross-sectional study. The research protocol was a questionnaire that allowed us to characterize the sample at a sociodemographic, academic, affective and sexual level. We included the Dating Violence Practices and Behaviors Scale (Dixe, Rodrigues, Freire et al., 2010) and the Sexual Sensations Search Scale (Carvalho \& Batista, 1998). Results: This is a mostly female sample (69.4\%). Regarding dating violence practices and behaviors, we found, in the dimensions under analysis, maximum values of 80.70 for psychological violence, 91.67 for stalking violence and sexual violence and 90.48 for physical violence. The results of the decision tree reveal that the search for sexual sensations is the variable that best predicts the degree of dating violence. Conclusion: Since violence in intimate relationships damages physical, psychological, sexual, reproductive, social, academic and professional development, with effects on people's health and well-being, both in the short, medium and long term, we propose awareness-raising actions in the school context promoted by experts and debated among peers.

Keywords: violence; dating; students; higher education; sexual sensations

\section{INTRODUÇÃO}

Segundo a Organização Mundial de Saúde (Organização Mundial de Saúde, 2010), a violência no namoro, refere-se a todo o comportamento que pode causar dano físico, sexual ou psicológico, abrangendo os casos de agressão física, a coação sexual, maltrato psicológico e comportamentos dominantes.

A violência no namoro é considerada como um tipo de violência interpessoal, existindo fatores de risco para a sua perpetração. Estes, podem ser agrupados em dimensões chave, nomeadamente problemas de saúde mental, atitudes de aceitação da violência nas relações de namoro, utilização de meios agressivos, comportamento agressivo para com os pares ou outros, uso de substâncias, comportamento sexual precoce, ter pares antissociais, idade, sexo e raça. Por outro lado, o facto de ser empático, ter boas notas, ter um QI verbal alto, ter uma relação positiva com a mãe e um sentimento de apego à escola, são referidos como fatores de proteção (Vagi, Rothman, Latzman, Tharp, Hall \& Breiding, 2013).

Já Dardis, Dixon, Edwards \&Turchik (2014), através de uma revisão da literatura sobre a perpetração da violência no namoro, referem que, na generalidade, muitos dos correlatos e preditores da perpetração se assemeIham entre sexos, como é o caso de o facto de ter testemunhado violência interparental, sofrer abuso em criança, abuso de álcool, papéis tradicionais do género e dinâmicas de poder relacional. No entanto, a perpetração de violência doméstica em mulheres jovens se relaciona mais com a interiorização de sintomas, nomeadamente a depressão, raiva e hostilidade, enquanto que a perpetração de violência no namoro em homens jovens está mais fortemente relacionada com o estatuto socioeconómico, nível de educação mais baixo, características de personalidade antissocial e uma maior duração do relacionamento.

A Organização Mundial de Saúde (2010) considera que este tem sido um problema de saúde pública desvaIorizado e mal percebido e acrescenta no se reporta aos efeitos na saúde que lhe estão subjacentes salientam-se as lesões corporais, a gravidez indesejada, 0 aborto, as complicações ginecológicas, as infeções sexualmente transmitidas, a síndrome de stresse pós-traumático, depressão e suicídios. Face à gravidade da problemática, esta Organização, recomenda a implementação de programas centrados na prevenção primária, com o foco nos jovens, em detrimento de programas de prevenção secundária. Neste âmbito, o IV Plano Nacional Contra a Violência Doméstica, referido por Santos (2014), acentua a necessidade de dar atenção à prevenção da vitimização de jovens e à violência simbólica, no que se refere às desigualdades de género, estreitamente relacionada com os processos de socialização. 
Muitas vezes a violência ocorre, aos olhos de quem a prática, como estratégia de resolução de conflitos. Assim, no seio das relações interpessoais amorosas, encontram-se frequentemente situações que abrangem agressões físicas, sexuais e/ou psicológicas, resultando em violência no namoro, um tema que tem suscitado 0 interesse da comunidade científica e de outros setores públicos, assumindo cada vez mais um lugar de destaque no discurso científico, político, jurídico, nos meios literários e nos meios de comunicação social (Guerreiro, Pontedeira, Sousa, Magalhães, Oliveira \& Ribeiro, 2014).

Nas relações de violência emerge o medo, a vergonha e a culpa por parte da vítima. Quando existe violência, o parceiro é física, sexual e verbalmente agressivo, não permite autonomia ao outro, controla tudo o que 0 outro faz, humilha-0, culpa o parceiro/a pelos seus atos, não permite o fim da relação, pressiona-0, impõe-se na relação sexual e tenta compensar os comportamentos violentos (Ferreira, 2013). Assim, um relacionamento violento pode desencadear nos jovens baixa autoestima, depressão, raiva, ansiedade, insucesso escolar, ideação suicida, perturbações alimentares, envolvimento em comportamentos sexuais de risco, consumo de substâncias e comportamentos de risco associados (Caridade, Saavedra \& Machado, 2012).

Apesar do impacto negativo da violência no namoro, muitas vítimas, particularmente os estudantes universitários, não procuram ajuda. Até à atualidade, a procura de ajuda tem-se resumido a uma forma de vitimização, apesar das evidências de violência interpessoal. Assim, os esforços desenvolvidos para incrementar a procura de ajuda por parte das vitimas, podem ser benéficos, pois permitem compreender os preditores de procura de ajuda, através de tipos de vitimização. No âmbito desta análise, os resultados do estudo desenvolvido por Ameral, Palm Reed \& Hines (2020), forneceram novas provas de sobreposição de barreiras de procura de ajuda entre tipos de violência, com por exemplo a crença da vítima de que 0 incidente não era suficientemente grave para procurar ajuda, entre os três tipos de vitimização. Assim, esta sobreposição de barreiras pode alertar para a necessidade de deligenciar esforços para a prevenção e delinear estratégias de intervenção para todos os tipos de vitimização.

Tendo em consideração o impacto que a violência no namoro tem na saúde pública, esta temática permanece ainda um pouco marginalizada nos discursos sociais e educativos e, em comparação com a violência marital, a vitimação de adolescentes tem merecido menor atenção por parte da comunidade científica (Ferreira, 2013). Todavia, a violência nas relações de namoro nos estudantes do ensino superior é cada vez mais comum e tem sido considerada um problema social significante, tornando-se, assim, um aspeto importante para um crescente número de estudos (Ávila, 2013).

\section{OBJETIVOS DA INVESTIGAÇÃO}

Determinar se a procura de sensações sexuais influencia as práticas e comportamentos de violência no namoro.

\section{AMOSTRA E PARTICIPANTES}

Trata-se de uma amostra não probabilística por conveniência constituída por 820 estudantes do ensino superior (média de idade de 20.48 anos. Dp=2.51).

\section{METODOLOGIA E INSTRUMENTOS UTILIZADOS}

Trata-se de um estudo quantitativo, descrito-correlacional e transversal. 0 protocolo de investigação foi um questionário que permitiu caracterizar a amostra a nível sociodemográfico, académico, afetivo e sexual. Incluímos a Escala de Práticas e Comportamentos de Violência no Namoro (Dixe, Rodrigues, Freire et al., 2010) e a Escala de Procura de Sensações Sexuais (Santos, Ferreira, Carvalho \& Ferreira, 2017). Na presente investigação foram salvaguardados os direitos dos participantes e os princípios éticos fundamentais. 0 tratamento estatístico foi processado através do programa SPSS versão 21.0 (2013) para Windows. 


\section{IMPACTO DA PROCURA DE SENSAÇÕES SEXUAIS NOS COMPORTAMENTOS DE VIOLÊNCIA NO NAMORO}

\section{RESULTADOS ALCANÇADOS}

\section{Caracterização sociodemográfica}

Em relação ao estado civil verifica-se que $57.2 \%$ são solteiros (com representatividade de para o sexo masculino de $62.9 \%$ e ao sexo feminino $54.7 \%$. Existe um claro predomínio de participantes que provêm de zona rural (55.6\%). Quanto ao ano de licenciatura que frequentam em função do sexo prevalecem os estudantes no $1^{0}$ ano (35.0\%). A quase totalidade da amostra (95.4\%) é apenas estudante.

\section{Relação entre a procura de sensações sexuais e violência no namoro}

A escala de Procura de Sensações Sexuais (validada para a população portuguesa por Santos, Ferreira, Carvalho \& Ferreira, 2017), é composta por dois fatores (F1 - Busca de novas sensações sexuais e F2 - Busca de novas experiências sexuais). Verificamos que a procura de experiências sexuais é o fator que apresenta a média mais elevada (49.21; Dp=30.091), por seu lado o fator procura de emoções e aventuras tem uma média de 41.04 ( $\mathrm{Dp}=24.326)$. A média encontrada na procura das sensações sexuais (escala global) é de 44.11 $(\mathrm{Dp}=23.960)$.

Quanto à violência no namoro, verifica-se que todas as dimensões de violência obtiveram um mínimo de 0.00, oscilando os valores máximos entre 80,70 (violência psicológica) 91,67 (violência stalking e violência sexual), tendo a violência física um máximo de 90.48 .

Observando a prática de comportamentos de violência no namoro em função das variáveis sociodemográficas e académicas, verifica-se que 0 sexo e 0 estado civil apresentam significância estatística, com valores de menor violência para o sexo feminino e estado civil de solteiro.

Foram realizadas regressões lineares múltiplas univariadas para estudar a relação entre variáveis. 0 método de estimação usado foi o "stepwise". Quanto à relação entre a procura de sensações sexuais e a violência física e sexual verificamos que existe significância estatística $(\mathrm{p}=0.000)$ para "Busca de novas sensações sexuais", com relação positiva $(r=0.149)$. Relativamente à relação entre procura de sensações sexuais e a violência stalking foram observadas diferenças estatísticas significativas $(\mathrm{p}=0.000)$ para "Busca de novas sensações sexuais" e "Busca de novas experiências sexuais", com relação positiva $(r=0.217)$ e $(r=0.179)$ respetivamente. Analisando a relação entre a procura de sensações sexuais e a violência psicológica verificamos significância estatística $(p=0.000)$ para "Busca de novas sensações sexuais" e "Busca de novas experiências sexuais", com relação positiva $(r=0.223)$ e $(r=0.155)$ respetivamente.

\section{DISCUSSÃO}

A violência no namoro consiste em todo o comportamento que pode resultar em dano físico, sexual ou psicológico, abarcando os casos de agressão física, a coação sexual, maltrato psicológico e os comportamentos dominantes (Organização Mundial de Saúde, 2010). Ela, é considerada um tipo de violência interpessoal, com fatores de risco para a sua perpetração, nomeadamente os problemas de saúde mental, atitudes de aceitação da violência nas relações de namoro, utilização de meios agressivos, comportamento agressivo para com os pares, comportamento sexual precoce, ter pares antissociais, idade e sexo. (Vagi et al., 2013).

Constatou-se que são os estudantes do sexo masculino os que manifestam mais o comportamento de violência no namoro, sobretudo a violência stalking e a violência psicológica. Estes resultados estão em concordância com os de Saavedra e Machado (2012), segundo os quais, existem diferenças significativas na violência perpetrada pelos estudantes do sexo masculino, em relação ao feminino. De acordo com os mesmos autores, a violência psicológica e a stalking são as mais evidentes, confirmando os dados apurados no presente estudo. Ao nível das relações de namoro, a violência stalking consiste num padrão de comportamentos de assédio persistentes, repetidos e indesejados, cujo objetivo é a vigilância e o controlo de uma pessoa alvo por parte de um stalker.

Os resultados revelam que o estado civil interfere nos comportamentos de violência no namoro, sendo os participantes que não se encontram numa relação de namoro que apresentam mais comportamentos de violência 
física e sexual. Nos restantes tipos de comportamentos de violência no namoro, os valores médios são mais elevados no grupo que se encontra numa relação, com destaque para a violência stalking, seguindo-se a violência psicológica. Mendes, Duarte, Araújo \& Lopes (2013). no seu estudo, refere que os estudantes deixaram transparecer que existe uma ideia comum de que a violência sexual acontecerá sempre que for ultrapassado um limite. Esse limite, refere-se sempre às situações em que não é respeitada a vontade de outrem.

A violência stalking não é, por si só, tida como intrusiva e ameaçadora, uma vez que, na opinião de Grangeia e Matos (2011), pode revestir-se de conotações positivas e lisonjeadoras para o alvo. Todavia, os mesmos autores admitem que esta se assume como uma fronteira ténue que separa, de um lado, a expressão de afetos culturalmente aceite e, do outro, 0 assédio e a perseguição de caráter intrusivo, que parece ter protelado a configuração do stalking como categoria de vitimação, cujas repercussões no bem-estar psicológico das vítimas são visíveis, sobretudo por parte de jovens que se encontram numa situação de namoro, o que foi corroborado com os nossos dados.

Importa também ressalvar a perspetiva de Mendes et al. (2013), onde referem que os estudantes consideraram que, nas relações de namoro, por serem mais fechadas, torna-se mais difícil detetar situações de violência. Não obstante, importa referir que, por vezes, numa relação de namoro se desvalorizam os primeiros sinais de violência, o que pode justificar os resultados encontrados no presente estudo, onde a violência psicológica e a stalking é perpetrada pelos que namoram.

A busca de sensações em contexto de atividades sexuais tem sido alvo de investigação, pois predispõe para a procura de sensações variadas, complexas e intensas, no âmbito da sexualidade, envolvendo algum risco (Santos, Ferreira, Duarte, Ferreira, 2017). No nosso estudo, a procura de sensações sexuais estabelece uma relação direta com a prática de violência stalking, sugerindo que quanto maior a procura de sensações sexuais maior é a probabilidade de violência stalking. Neste âmbito, o estudo de Basile e Hall (2011) revelou que a violência stalking está altamente correlacionada com a procura de sensações sexuais e com os outros tipos de violência, devendo ser vista como um tipo de violência específico e não como um subtipo de abuso psicológico. Assim, fazendo-se referência a Caridade e Machado (2010), importa reforçar o pressuposto de que a violência stalking, em muitas situações, não é percebida pela vítima durante ou após a relação de namoro, estando intimamente relacionada com o isolamento que é imposto pelo(a) parceiro(a). A dificuldade em reconhecer que se é vítima de stalking prende-se com a o facto de se acreditar que a violência é uma forma de demostrar 0 amor, passando a verse a violência como algo natural.

Deste modo, pode dizer-se que as evidências empíricas encontradas no presente estudo se revelam concordantes com a literatura, sendo evidente a existência de comportamentos de violência no namoro entre os estudantes do ensino superior, com particular destaque para a violência psicológicas.

\section{CONCLUSÕES}

A violência no namoro é um problema grave, não só pela sua significativa prevalência, mas também pelas suas consequências ao nível da saúde física e mental.

São os estudantes do sexo masculino que manifestam mais comportamentos de violência no namoro, sobretudo a violência stalking e a violência psicológica. Verificou-se que, quanto maior for a procura de sensações sexuais, maior a prática de comportamentos de violência física, sexual, psicológica e stalking.

Face aos resultados, ganha toda a relevância a promoção de intervenções mais adequadas às necessidades dos estudantes, demonstrando-Ihes as consequências que a violência no namoro acarreta para o seu bem-estar físico e psicológico. Nestas ações de sensibilização, devem também ser abordadas temáticas relativas às crenças associadas à violência no namoro e atitudes que legitimam a violência.

0 esforço preventivo, em contexto escolar, beneficiará com uma articulação ativa entre a escola e a comunidade. Também, as instituições de ensino superior devem estar sensibilizadas para esta problemática e serem um potencial protetor de apoio social e comunitário, desenvolvendo programas/intervenções para diversas populações. 


\section{IMPACTO DA PROCURA DE SENSAÇ̃̃ES SEXUAIS NOS COMPORTAMENTOS DE VIOLÊNCIA NO NAMORO}

De igual forma, seria ainda importante a utilização das novas tecnologias digitais, para elaborar e colocar em prática programas online, geridos por profissionais, sobre 0 impacto da violência no namoro, permitindo, desta forma, o esclarecimento de dúvidas.

\section{AGRADECIMENTOS}

Este trabalho é financiado por Fundos Nacionais através da FCT - Fundação para a Ciência e a Tecnologia, I.P., no âmbito do projeto Refạ UIDB/00742/2020. Agradecemos adicionalmente à Unidade de Investigação em Ciências da Saúde: Enfermagem (UICISA: E) e ao Politécnico de Viseu pelo apoio prestado.

\section{REFERENCIAS BIBLIOGRÁFICAS}

Ameral, V., Palm Reed, K. M., \& Hines, D. A. (2020). An analysis of help-seeking patterns among college student victims of sexual assault, dating violence, and stalking. Journal of Interpersonal Violence, 35(23-24), 53115335. doi:10.1177/0886260517721169.

Ávila, A. C. C. (2013). Violência entre parceiros íntimos: Análise da relação com o consumo de drogas e álcool numa amostra de estudantes do ensino superior [Dissertação de mestrado, Universidade de Coimbra]. ESTUDO GERAL: Repositório científico da UC. https://estudogeral.uc.pt/handle/10316/24851

Basile, K., \& Hall, J. (2011). Intimate partner violence perpetration by court-ordered men: Distinctions and intersections among physical violence, sexual violence, psychological abuse, and stalking. Journal of Interpersonal Violence, 26(2), 230-253. doi: 10.1177/0886260510362896.

Caridade, S., \& Machado, C. (2010). Novas formas de vitimação criminal. Psiquilibrios edições.

Caridade, S., Saavedra, R., \& Machado, C. (2012). Práticas de prevenção da violência nas relações de intimidade juvenil: Orientações gerais. Análise Psicológica, 30(1-2), 131-142. http://repositorio.ispa.pt/handle/10400.12/3411

Dardis, C. M., Dixon, K. J., Edwards, K. M., \& Turchik, J. A. (2015). An examination of the factors related to dating violence perpetration among young men and women and associated theoretical explanations: A review of the literature. Trauma Violence Abuse, 16(2), 136-52. doi: 10.1177/1524838013517559.

Dixe, M., Rodrigues, A. L., Freire, C., Rodrigues, G., Fernandes, M., \& Dias, T. (2010, Fevereiro 4-6). A violência de género na relação de namoro em estudantes do ensino superior: Práticas e comportamentos de violência [Comunicação]. VII Simpósio Nacional de Investigação em Psicologia, Braga. https://iconline.ipleiria.pt/handle/10400.8/334

Ferreira, J. P. (2013). Stalking como forma de violência nas relações de namoro [Dissertação de mestrado, Instituto Superior de Ciências da Saúde Egas Moniz, Almada). Repositório Comum. https://comum.rcaap.pt/handle/10400.26/6243

Grangeia, H., \& Matos, M. (2011). Da invisibilidade ao reconhecimento do stalking. In A. I. Sani, Temas de vitimologia: Realidades emergentes na vitimação e respostas sociais (pp. 61-84). Almedina.

Guerreiro, A., Pontedeira, C., Sousa, B., Magalhães, M. J., Oliveira, E., \& Ribeiro, P. (2014). Intimidade e violência no namoro: Refletir a problemática nos/as jovens. Cescontexto, 10, 14-26.

https://repositorioaberto.up.pt/bitstream/10216/78885/2/115603.pdf

Mendes, J., Duarte, M., Araújo, P., \& Lopes, R. (2013). Violência e relações de intimidade no ensino superior em Portugal: Representações e práticas. Teoria \& Sociedade, 21(2), 87- 112. http://hdl.handle.net/10316/36367

Organização Mundial de Saúde. (2010). Prevenção da violência sexual e da violência pelo parceiro íntimo contra a mulher: Ação e produção de evidência. Organização Pan-Americana da Saúde. http://apps.who.int/iris/bitstream/10665/44350/3/9789275716359_por.pdf

Saavedra, V., \& Machado, C. (2012). Programas de prevenção primária da violência nos relacionamentos íntimos: Da prática internacional à prática nacional. Revista de Psicologia da Criança e do Adolescente, 4(1), 65-93. http://repositorio.ulusiada.pt/bitstre-am/11067/962/1/rpca_v4_n1_4.pdf 
Santos, A. S. D. (2014). Violência no namoro em jovens que vivenciam pobreza e exclusão social [Dissertação de mestrado, Instituto Universitário da Maia). Repositório Científico do ISMAI. https://repositorio.ismai.pt/handle/10400.24/343

Santos, M. J. 0., Ferreira, E. M. S., Duarte, J. C. D., \& Ferreira, M. M. C. (2017).Adaptação portuguesa e validação da Sexual Sensation. Revista de Enfermagem Referência, 4(15), 11-20. https://doi.org/10.12707/RIV17054

Vagi, K. J., Rothman, E. F., Latzman, N. E., Tharp, A. T., Hall, D. M., \& Breiding, M. J. (2013). Beyond correlates: A review of risk and protective factors for adolescent dating violence perpetration. Journal of Youth Adolescence, 42, 633-649. https://link.springer.com/content/pdf/10.1007/s10964-013-9907-7.pdf 
